\title{
La filosofía como humanización de la persona: Entrevista al maestro Daniel Herrera Restrepo
}

\author{
Philosophy as humanization of the person: \\ Interview to Master Daniel Herrera Restrepo \\ A filosofia como humanização da pessoa: \\ Entrevista com o Mestre Daniel Herrera Restrepo
}

Fecha de entrega: 26 de julio de 2013 Fecha de evaluación: 15 de noviembre de 2013 Fecha de aprobación: 20 de diciembre de 2013

Tatiana Castañeda y Fernando Alba*

\section{Resumen}

La siguiente entrevista busca servir de homenaje a uno de los filósofos colombianos que más conoce y comprende el pensamiento de Husserl, quien con su trabajo ha motivado la difusión de la fenomenología en Colombia. Hace referencia a la importancia que tuvo el contacto de Daniel Herrera con los archivos de Husserl, el haber podido participar en grupos de estudio de renombre, así como la comprensión del mundo

Tatiana Castañeda López es Licenciada en Filosofía y Letras de la Universidad Santo Tomás. Magister en Educación de la Universidad Pedagógica Nacional. Coordinadora de prácticas de la Facultad de Filosofía de la Universidad de San Buenaventura. Correo electrónico: tatifilo@hotmail.com Fernando Alba es Licenciado en Filosofía de la Universidad de San Buenaventura. Contacto: nelsonalba@hotmail.com Miembros del Grupo de Investigación en Filosofía Contemporánea, DEVENIR. Facultad de Filosofía, Universidad de San Buenaventura. 
de la vida asumido como un concepto capaz de vincular la vivencia cotidiana con el modo original como llegamos a explicarnos a nosotros mismos, lo cual sirve de base para identificar la filosofía de nuestro continente, ya que esto no se desliga del quehacer crítico sobre el orden dado y orientador entorno a las nuevas maneras de experimentar nuestra vida a partir de las propias diferencias.

Palabras clave: Husserl, mundo de la vida, filosofía latinoamericana, fenomenología.

\section{Abstract}

The following interview seeks to pay tribute to one of the Colombian philosophers who best knows and understands the thinking of Husserl, who with his job has motivated the spreading of phenomenology in Colombia. It refers to the importance of the contact Daniel Herrera had with Husserl's files, having been able to participate in renowned study groups, as well as in the understanding of the life-world as a conception capable of connecting daily life with the original form we got to explain ourselves, which serves as basis to identify the philosophy of our continent, since this does not separate from the critical task on the given and orienting order around the new forms of experiencing our lives from our own differences.

Keywords: Husserl, life-world, Latin America philosophy, phenomenology 


\section{Resumo}

A entrevista a seguir procura servir de homenagem a um dos filósofos colombianos que mais conhece e compreende o pensamento de Husserl, quem com o seu trabalho tem motivado a difusão da fenomenologia na Colômbia. Refere-se à importância que teve o contato de Daniel Herrera com os arquivos de Husserl, e ter participado em grupos de estudo de renome, assim como a compreensão do mundo da vida apropriado como um conceito capaz de vincular a vivência do dia a dia com o modo original chegarmos a explicarmos a nós mesmos, que é a base para identificar a filosofia do nosso continente, devido a que isto não se desliga do quefazer crítico sobre a ordem dado e orientador abordado às novas maneiras de experimentar nossa vida a partir das próprias diferenças.

Palavras-chave: Husserl, mundo da vida, a filosofia latino-americana, fenomenologia. 
El maestro Daniel Herrera Retrepo es hoy por hoy uno de los filósofos colombianos más importantes y a quien le debemos el trabajo más significativo en el país en el ámbito de la fenomenología, particularmente en el estudio y la recepción de la fenomenología de Husserl. Autoridad en sus reflexiones acerca de la promoción y desarrollo del pensamiento filosófico en Colombia, este destacado académico e intelectual ha dedicado gran parte de su vida a la filosofía. Recientemente su obra ha sido declarada patrimonio de la humanidad por la Unesco. Con el carisma y amabilidad que siempre lo han caracterizado, sostuvo una amena conversación sobre diversos aspectos de su trayectoria intelectual, su extensa producción bibliográfica y sus ideas críticas en lo referido a los presupuestos de la fenomenología, su comprensión de la filosofía y los procesos de normalización de la misma en el país.

Tatiana Castañeda y Fernando Alba: ¿Qué significó conocer los inéditos de Husserl en los archivos de Lovaina para su trabajo como estudioso de la fenomenología?

Daniel Herrera: A partir de 1951 se empiezan a publicar los inéditos de Husserl, lo cual fue un impacto novedoso para la filosofía del momento, pues se conocía a Husserl como un idealista. Su comprensión del mundo de la vida supuso todo un cambio en su lectura, es más, influyó a muchos filósofos de la época. Debo señalar que los archivos de Husserl fueron mi mayor escuela. Imagínese usted lo que para un estudiante significaba asistir a los archivos. Recuerdo bien las reuniones privadas los martes en la noche, convocadas por Herman Van Breda para analizar y comentar los inéditos de Husserl; puedo decir que asistí a todas. Recuerdo una en particular: estaban Emmanuel Lévinas, Merleau-Ponty, Gabriel Marcel y Jean Paul Sartre. Lévinas un creyente judío, Marcel un católico, Sartre un ateo declarado, todos interpretando un texto de Husserl, era extraordinario. Tuve acceso a mucha de la correspondencia de los archivos. Por ejemplo, recuerdo mucho una carta de Étienne Gilson a Van Breda en la que la expresaba su fuerte interés por la fenomenología, pero en la que se lamentaba por estar muy viejo para investigar en los archivos.

T. C. y F. A.: Es evidente que su trabajo filosófico supone un importante desarrollo de varios presupuestos de Husserl, particularmente del periodo en el cual el filósofo comprende la fenomenología como la ciencia del mundo de la vida. En su extensa obra usted tematiza el mundo de la vida y a partir de él revisa críticamente la realidad colombiana. ¿Qué piensa al respecto de este trabajo suyo sobre el mundo de la vida en nuestro país? 
D. H.: Una experiencia personal: cuando llegue al país en 1962 traía muy presente la idea de mundo de la vida y quise saber hasta qué punto los pensadores colombianos habían vivido su mundo, lo habían pensado, lo habían asimilado. Empecé a investigar en diversas bibliotecas de conventos, sus principales obras del siglo XVII y XVIII, y en dicha búsqueda encontré a José Félix de Restrepo: la mayoría de su obra estaba inédita. Debo decir que no publiqué el texto que hubiese querido sobre el desarrollo de la filosofía en Colombia desde el punto de vista de la reflexión de nuestra realidad. Mi intención no era publicar una bibliografía pero la Facultad de Filosofía de la Universidad del Valle insistió en publicar la bibliografía que había recogido años atrás, mas no quería pues estaba incompleta (Herrera, 1975, p. 248). Con el paso de los años la bibliografía se convirtió en un texto de consulta fundamental en la Universidad Santo Tomás puesto que sugería la existencia de un pensamiento colombiano.

T. C. y F. A.: Usted regresa al país con los análisis que Husserl realiza sobre la actualidad europea en mente. ¿Pensaba hacer extensivos dichos análisis a la realidad nacional o latinoamericana?

D. H.: Precisamente en "Sobre la posibilidad de una filosofía latinoamericana" realicé una exploración de ese tipo (Herrera, 2009). Era evidente el tratamiento dado por Husserl a la cuestión de la existencia de una unidad espiritual europea en Crisis (Husserl, 1991, pp. 323-358). Sin perder de vista estos presupuestos y la forma como Husserl realiza el análisis, me vi avocado a explorar la existencia de una filosofía colombiana y la de una latinoamericana, con los resultados ya conocidos por todos. Sin embargo y a pesar de negar la posibilidad de una filosofía latinoamericana o colombiana, reconocí ciertos rasgos y formas de comprensión de nosotros mismos bastante originales que no responden exclusivamente a una comprensión de la razón como razón demostrativa. Ahora bien, debo decir que, por ejemplo, en literatos como Jorge Isaacs o García Márquez fue posible encontrar expresiones bien particulares, rasgos de comprensión propios del ser colombiano, y ese fue un factor determinante en el momento de establecer quiénes han pensado nuestra realidad y cómo la han pensado. Nosotros los latinoamericanos argumentamos desde el deseo, la experiencia y, por último, desde el logos griego; son perspectivas distintas, otros tipos de racionalidad. No es un secreto para nadie cómo opera un europeo con su logos griego y cómo lo hace un latinoamericano con su forma de racionalidad propia y a su vez con el mismo logos griego. ¿ No era 
acaso Aristóteles quien ubicaba en último término la capacidad demostrativa como forma de conocer la realidad? Esta capacidad demostrativa -manifiesta en la frase "esto es así y no puede ser de otra manera"- se potencia considerablemente en la modernidad con el racionalismo.

T. C. y F. A.: Maestro, entre las múltiples concepciones que usted ha planteado respecto de la filosofía, tal vez la más conocida sea: "la filosofía es una estructura lógica y coherente de los diversos aspectos que definen una sociedad orientados a reflexionar de manera sistemática, crítica y prospectiva sobre las diversas prácticas del hombre frente a la realidad" (Herrera, 2009, p.17). Atendiendo a la misma definición, ¿cómo se sitúa la labor del filósofo en una sociedad como la colombiana?

D. H.: Pregunta difícil, pues nosotros mismos queremos llegar a una comprensión de la realidad en sí misma, operamos como no latinoamericanos, desatendiendo a la forma como usualmente operamos ante nuestra realidad próxima. Quien ha tenido la oportunidad de conocer otra cultura puede dar cuenta de ello. Por ejemplo, la forma de proceder de la cultura alemana difiere enormemente de la nuestra. De esta manera, la labor del filósofo no puede ser otra sino la de llevar a los otros a pensar en sus realidades, a cómo comprenden su tiempo y se comprenden a sí mismos como sujetos situados en un contexto y en una sociedad determinada. Es necesario emprender nuevas rutas de exploración sobre cómo nos comprendemos, desplazando considerablemente la razón demostrativa, abandonando todo dogmatismo.

T. C. y F. A.: Teniendo en cuenta que, según usted, el filósofo está llamado a ser la conciencia crítica de la sociedad, en su larga trayectoria como filósofo, ¿quiénes, considera, han realizado esta labor en el país?

D. H.: Es evidente el esforzado trabajo de muchas personas por el desarrollo de la filosofía en Colombia. Tal es el caso de Guillermo Hoyos quien desafortunadamente falleció hace poco. Guillermo siempre estuvo pensando nuestra realidad social desde muchas perspectivas, como la filosofía, la sociología, la pedagogía, la política. Otros, como Cayetano Betancur, Luis Eduardo Nieto Arteta, Rafael Carrillo, Danilo Cruz Vélez, también lo hicieron a su manera. Estos últimos, lo hicieron en particular con la fundación del Instituto de Filosofía, hoy Departamento de Filosofía de la Universidad Nacional de Colombia. Aunque la historia del Instituto también tuvo sus inconvenientes. Recuerdo la desafortunada participación de Víctor Frankl en el estancamiento del Instituto de Filosofía para ese tiempo. Él mismo convenció 
al presidente Laureano Gómez de la necesidad de retomar los estudios tomistas en filosofía, porque, al parecer, nosotros no habíamos tenido Edad Media, lo cual supuso un cambio fundamental en las orientaciones del Instituto en ese momento. Todo ello repercutió incluso en la clausura temporal de la revista Ideas y Valores. Ahora, lo positivo de dicha clausura fue el necesario origen de la revista Mito en 1955, publicación que se convirtió en un importante escenario expositivo para la filosofía contemporánea en Colombia: Sartre, Husserl, Scheler, Heidegger.

T. C. y F. A.: A este respecto, ¿cómo ha sido la relación del rol del filósofo con los procesos de normalización y de institucionalización de la enseñanza de la filosofía en Colombia?

D. H.: Esta relación ha sido bien compleja, cambiante en su dinámica, pero efectivamente prolífica. Hay que distinguir primero una generación de filósofos, la del Rosario, entre 1900 y 1930, caracterizada por un débil conocimiento del tomismo. No obstante, son bien significativas las cartas que como ministro de educación escribía Luis López de Mesa a Julio Enrique Blanco, invitándolo a la creación de una facultad de filosofía. Se desatacan a su vez otros pensadores como Baldomero Sanín Cano, Carlos Arturo Torres, Agustín Nieto Caballero. Creo que en sentido estricto Julio Enrique Blanco fue el único filósofo en Colombia antes de 1930. Entre 1930 y 1960 se da una segunda generación, la de los fundadores del Instituto de Filosofía que mencionaba anteriormente.

Hacia 1930, la Facultad de Derecho de la Universidad Nacional de Colombia le da una importancia capital a la filosofía. Hasta el punto de que a mucha gente que no le interesaba para nada el derecho cursaban sus estudios en esta facultad, tal es el caso de Danilo Cruz Vélez, Rafael Carrillo y Luis Eduardo Nieto Arteta. Es en este contexto que yo regreso de Europa, participo en la fundación de la Facultad de Filosofía de la Universidad de San Buenaventura y Franciscanum sale a la imprenta ya que solo existía en mimeógrafo. Para la época Ideas y Valores y Franciscanum eran las únicas dos revistas de filosofía en el país.

T. C. y F. A.: La fundación del Instituto de Filosofía de la Universidad Nacional se suele asumir como referente histórico para hablar de procesos de normalización de la filosofía en Colombia. ¿Esto no sería para usted más bien una institucionalización de la enseñanza de la filosofía y no propiamente normalización? 
D. H.: Por supuesto, hay que decir que a la hora de la verdad la filosofía se ha dado en Colombia esencialmente en un nivel universitario, es decir, si no se está enseñando filosofía uno se puede morir de hambre.

Francisco Romero denominó normalización al proceso vivido en las primeras décadas del siglo XX, en virtud del cual el filosofar empezó a ser reconocido como función ordinaria dentro de la cultura de los países latinoamericanos. Usted distingue varios factores que inciden directamente en la normalización filosófica: órganos de difusión, programas, revistas, foros de filosofía, bibliotecas, movilidad estudiantil, idiomas. Al parecer buena parte de estos se han desarrollado durante los últimos años en el país. No obstante, algunos filósofos colombianos consideran que la normalización todavía no es un hecho. ¿Qué hace falta?

Yo creo que sí se puede hablar de cierta normalización de la filosofía en algunas facultades y departamentos de filosofía. Lastimosamente no en todos, pero en su gran mayoría hay un reconocimiento de la actividad filosófica con todo lo que ello implica. Ahora bien, si se habla de normalización como reconocimiento de la función ordinaria de la cultura, la negativa como respuesta es inminente, en esta perspectiva es mucho más difícil de afirmar.

T. C. y F. A.: De esta manera, ¿la filosofía debe restringirse a los filósofos de formación y a las facultades de filosofía o, en cambio, debe democratizarse en cuanto a su enseñanza en las facultades de humanidades y en la educación secundaria? ¿Esta democratización de la enseñanza supone algún tipo de riesgo en relación con la profundidad y sistematicidad con la que se debe asumir la enseñanza de la filosofía?

D. H.: Actualmente en algunas universidades las facultades de filosofía son realmente facultades de filosofía, es decir, asumen la enseñanza de la filosofía como un presupuesto transversal para la formación de toda clase de disciplinas. Otras universidades, por su parte, no han asumido dicha tarea, para ello es necesaria una buena biblioteca, rica en textos clásicos de la filosofía, en lenguas originales. De manera que sea posible que los profesores guíen a sus estudiantes en la lectura y en el análisis crítico de autores clásicos de la filosofía. Todo lo anterior mediante el desarrollo sistemático de seminarios y demás actividades investigativas como las tesis de grado y la participación en eventos de carácter filosófico. 
La democratización de la enseñanza de la filosofía en las facultades de humanidades es una exigencia propia de la misma filosofía. Esta supone que cualquier estudiante, sin importar su tipo de carrera, pueda acercarse a los conceptos, autores y problemas fundamentales de la filosofía. Igual, sigo creyendo que la cuestión varía dependiendo del tipo de universidad que ofrezca las humanidades.

T. C. y F. A.: Sabemos que habla a partir de su experiencia formativa en Europa. Precisamente, en la entrevista que usted concedió a Salazar Ramos señala que quería estudiar inicialmente sociología en la Universidad de Lovaina (Salazar, 1986, pp. 11-35). En ese tránsito conoció a Camilo Torres. ¿Qué recuerdos tiene de esos años?

D. H.: En efecto, en Lovaina, cuna de los mayores estudios en sociología para la época, había un albergue de sacerdotes estudiantes, entre los cuales dos eran colombianos: Gustavo Pérez, también estudiante de sociología, y Camilo. En ese ambiente académico de Lovaina, Camilo se apasionó por los problemas sociales de Colombia, organizaba reuniones cada quince días para hablar sobre la situación del país, con el paso del tiempo las reuniones fueron tomando fuerza hasta el punto de invitar profesores especialistas para debatir con los asistentes. Inclusive alcanzamos a recibir ayuda de organizaciones extranjeras. El alma de todo eso era Camilo. Es esa época en que surge la vocación de Camilo. Qué fuerte la imagen de su asesinato, yo estuve con él en su despedida, antes de partir al monte, fuimos íntimos amigos, así la mayoría no estuviera de acuerdo con sus ideas políticas. A Camilo igual le tocaba irse pues estaba oficialmente condenado a muerte, a mí me dio duro lo que pasó. Una anécdota: recuerdo que Camilo se llevó a vivir a la mamá a Bélgica; él vivía en Bruselas a veinte minutos de Lovaina. En una ocasión estábamos con su mamá en su casa y una señora, que estaba de visita, le preguntó si existían semáforos de donde ella venía. La mamá de Camilo le responde "semáforos [...] déjeme ver, no, allá los pobres van en burros y los ricos en caimanes con cintas".

T. C. y F. A.: Retomando su comprensión de la filosofía, usted señala que como tarea "la filosofía está llamada a recuperar las diversas estructuras fundamentales y operantes de la existencia humana en su vida cotidiana, gracias a las cuales creamos ciencia, técnica, política, relaciones sociales, instituciones, historia; en fin, cultura" (Herrera, 2009, p. 306). Si la filosofía se constituye en la nota esencial del eidos de toda cultura, es decir, del ethos. Cuando afirma que no existe algo asíllamado filosofía latinoamericana, filosofía colombiana o incluso filosofía en Colombia, ¿a su vez niega la existencia de una identidad cultural colombiana o latinoamericana? 
D. H.: No. Precisamente en "Sobre la posibilidad de una filosofía latinoamericana" señalo que la filosofía nuestra debe asumir como tarea la explicitación de nuestra identidad. Aclaremos, la ausencia de una filosofía colombiana no supone la no existencia de una identidad cultural colombiana. Igual, ese es un problema bien serio pues en realidad no existe una identidad. Dígame: ¿qué tiene en relación un cachaco con un costeño? Nada. De modo que somos simplemente grupos sociales diferentes, cada uno con sus modos de ser distintos. No más pensemos en el lenguaje, sus usos y su polisemia cambiante de región en región.

T. C. y F. A.: A este propósito usted señalaba la necesidad de establecer una "crítica de la razón latinoamericana", acompañada de una explicitación de su lógica y de la determinación de sus categorías, las cuales permitirían elevar a un nivel conceptual nuestras vivencias específicas. Treinta años después de dicha propuesta, ¿cuál es el estado actual de la cuestión?; ¿qué opinión le merece la tarea realizada por Santiago Gómez-Castro en su obra Crítica de la razón latinoamericana?

D. H.: Debo reconocer que hay autores que han tratado de abordar la empresa y le han dado continuidad. Creo que se ha emprendido desde varios campos la crítica de la razón latinoamericana y colombiana aunque me parece que aún se nos ha dificultado la explicitación de su lógica. En todo caso es algo que se debe hacer necesariamente para podernos comprender, para proyectarnos en el mundo de la vida. Hasta que esto no suceda difícilmente podremos tener una filosofía propia en sentido estricto, así como tampoco un filósofo como conciencia crítica de la sociedad. Ahora bien, hay que tener cuidado con el uso de la expresión "crítica de la razón": tiene un peso enorme y serias implicaciones, recordemos su espíritu kantiano, por no decir moderno. Yo tendría mis reservas en el momento de hablar de una crítica de la razón latinoamericana por cuanto supone una posición paradójica: yo empleé inicialmente la expresión para cuestionar la existencia de un pensamiento latinoamericano y colombiano, era una imagen. Resulta paradójico realizar una exploración de este tipo con una categoría clásica de la filosofía para dar cuenta del pensamiento latinoamericano. Por su parte, Santiago Gómez-Castro hace un trabajo admirable en la medida que expone de forma analítica el estado actual de la cuestión y actualiza el debate para las jóvenes generaciones.

T. C. y F. A.: ¿Cuál es la relación de dicha empresa con la posmodernidad?; ¿no fue usted uno de los primeros en afirmar la necesidad de ampliar el concepto de razón, mucho tiempo antes que lo hicieran los llamados autores posmodernos? 
D. H.: No creo que sea del todo así. Sin embargo, hay una referencia importante en Hombre y filosofía que vale la pena mencionar (Herrera, 1970, p. 95). En esta obra, hago ese llamado a ampliar el concepto de razón. Como lo mencioné en algún momento, mi interés por saber cuáles autores habían pensado de alguna manera la realidad colombiana me llevó a explorar el pensamiento colombiano. Pude identificar notas características bien particulares de nuestras formas de racionalidad, es decir, de actuar y de comprender cómo somos. En fin, toda esta empresa de la crítica de la razón latinoamericana está estrechamente ligada a la necesidad de ampliar el concepto de razón y en este aspecto puede haber puntos en común con algunos de esos autores posmodernos. Ahora bien, en "Posmodernidad: ¿Ruptura con la Modernidad?" tomo distancia de ellos en puntos que considero esenciales como el de la crítica que hacen al universalismo, por nombrar solo alguno (Herrera, 2009, pp. 181-196). En todo caso yo reconozco que los análisis de estos autores generan la apertura a otras dimensiones de la racionalidad y del individuo que vale la pena explorar.

T. C. y F. A.: En varios de sus textos, usted refiere la necesidad de crear mediante la educación y la formación en filosofía una mentalidad secularizada ilustrada en los ciudadanos. Atendiendo al estado actual de la filosofía y de su enseñanza en el país ¿cree que dicha educación y formación se está impartiendo en Colombia?

D. H.: No me atrevería a dar una afirmación rotunda pues no conozco del todo el tema en cuestión. Considero que se ha hecho un trabajo importante pero es innegable que la filosofía aún no ha tocado ciertas fibras de la sociedad, o bueno, por lo menos del ciudadano del común. Estamos lejos de afirmar que la filosofía sea una realidad para nosotros, que sea una práctica cotidiana que se viva desde los primeros años de los ciudadanos en el hogar. Y no se trata de un asunto secundario, no, el hogar es un aspecto relevante en la formación de individuos con una mentalidad secularizada, en últimas, de ciudadanos activos en una democracia.

T. C. y F. A.: ¿Acaso el conflicto interno colombiano no cuestiona severamente la solidez del proyecto democrático al que se le ha apuntado tanto desde hace más de un siglo?

D. H.: Es evidente que no existe un proyecto democrático sólido en nuestro país. Hay y ha habido intentos, muchos de ellos en la educación, colegios y universidades, en los que se asume seriamente la formación democrática de los individuos, lo cual 
está encaminado a la construcción de sociedades democráticas donde los individuos mantienen relaciones fraternas y sólidas.

T. C. y F. A.: Maestro, a lo largo de sus numerosos escritos e intervenciones usted siempre se ha mostrado como un filósofo crítico: niega la existencia de una filosofía latinoamericana y colombiana, pone en entre dicho el tema de la identidad cultural colombiana, cuestiona la solidez de los proyectos democráticos emprendidos en la historia reciente del país. Ahora, del mismo modo usted por ejemplo comprende la democracia como un valor ético y social en construcción. ¿Cómo se hizo un filósofo crítico pero a su vez tan propositivo?

D. H.: Habría mucho que decir al respecto. Mi padre creo que tiene mucho que ver en eso. Él fue un ateo, liberal radical del siglo XIX, abogado, de padres campesinos. Fue amigo íntimo del presidente López Pumarejo, siempre mostró un interés particular por las causas y los movimientos sociales, las discusiones no se hacían esperar pero siempre en un ambiente de tolerancia y respeto. En fin, creo que todo el ambiente de mi casa me acostumbró a los temas políticos y sociales, los cuales fueron despertando en mí un interés particular con el pasar de los años.

T. C. y F. A.: Al parecer existen varios puntos de encuentro entre su trabajo y el del profesor Guillermo Hoyos, como la formación kantiana, la fenomenología de Husserl, la tematización del mundo de la vida, el llamado a formar una conciencia democrática como proyecto prioritario de los procesos educativos, en últimas, una visión crítica de la totalidad de los fenómenos sociales desde una perspectiva filosófica, prospectiva y coherente. Además de la participación en eventos decisivos para la historia reciente del país como la constituyente y los diálogos del gobierno con el M-19, el EPL y la Unión Patriótica, entre otros. Si estos son los puntos de encuentro, ¿cuáles serían los puntos de disenso?

D. H.: No podría hablar de disenso, si bien su filosofía estuvo fuertemente influenciada por el pensamiento de Habermas. Nuestras diferencias son sutiles, cosas de segundo orden, simplemente detalles. Estoy de acuerdo con los puntos que acertadamente señalan. Nuestras trayectorias fueron paralelas. Me enorgullecen esas palabras que escribiría Guillermo en la presentación de La responsabilidad del pensar, un sentido homenaje que le rendimos con varios especialistas de todo el mundo: "como lo señala mi colega Daniel Herrera Restrepo fundador de la fenomenología en Colombia" (Rocha de la Torre, 2008). Con Guillermo teníamos una amistad 
cercana, constantemente nos encontrábamos en diversas actividades, compartimos muchos intereses.

T. C. y F. A.: ¿Cuál cree usted que sea el mayor legado de Guillermo Hoyos para la filosofía en Colombia?

D. H.: Con ocasión del Segundo Coloquio Latinoamericano de Fenomenología escribí un texto titulado "Nuestra responsabilidad social: Una reflexión a partir de Husserl" (Herrera, 2003). Debo decir que aunque no mencioné a Guillermo en ningún lugar, el texto fue escrito pensando en él. Allí Husserl considera que la filosofía no es una profesión [Beruf] que no tiene su horario de trabajo de ocho a seis, sino que es una vocación [Berufung], los filósofos son los representantes vocacionales del espíritu de la razón. Lo singular de Guillermo Hoyos es que no era un autor de libros, sino de conferencias. Guillermo asumió la filosofía como un medio para humanizar plenamente las dimensiones fundamentales de la existencia humana: lo político, lo ético, lo social y lo educativo. Antes que ser profesor Guillermo era un formador. Él se interesó desde la filosofía por muchos problemas sociales del país, incluso interviniendo directamente en ciertas problemáticas: conformó una Comisión que estuvo en negociaciones con las FARC en Casaverde. Además, Guillermo sostuvo relaciones con importantes políticos, fue un filósofo que constantemente trató de reducir la brecha entre la filosofía y diversos sectores de la sociedad colombiana, es más, en la misma filosofía siempre propuso vías de diálogo con varias disciplinas de las ciencias humanas.

T. C. y F. A.: Usted como muchos de su generación ha contribuido en la apertura de un espacio para una actividad filosófica que supera la actividad de la enseñanza. ¿Cómo avizora el relevo generacional respecto a su trabajo en la formación de discípulos que le den largo aliento a la fenomenología?

El panorama es alentador y creo que hay gente comprometida que trabaja arduamente para darle largo aliento al trabajo que yo y otros tantos de mi generación iniciamos décadas atrás. Solo por citar algunos nombres y omitiendo otros tantos: Germán Vargas Guillen (Universidad Pedagógica Nacional), Miguel Ángel Villamil (Universidad de San Buenaventura), Pedro Juan Aristizábal (Universidad Tecnológica de Pereira), Iván Moreno en Manizales, Juan Manuel Cuartas (Universidad EAFIT), Ángela María Estrada (Universidad de los Andes), Mónica Marcela Jaramillo (Universidad Industrial de Santander), Carlos Eduardo Maldonado (Universidad Industrial de 
Santander), Julio Cesar Vargas Bejarano (Universidad del Valle), Leonardo Verano Gamboa (Universidad del Norte).

T. C. y F. A.: ¿Cuál es el significado para usted del hecho de que la Unesco declarara su obra patrimonio de la humanidad?

D. H.: Es un orgullo para mí todo este reconocimiento. Estoy muy agradecido con la Universidad de Memphis, pues, en sus archivos sobre la fenomenología de Husserl, conservan buena parte de mis escritos. A su vez, con las universidades que llevaran a cabo la actualización de la plataforma de Memphis: Universidad de Antioquia, Universidad Industrial de Santander y la Universidad Pedagógica Nacional.

T. C. y F. A.: ¿Cuál es la importancia de la filosofía para su vida?

D. H.: Toda. Hasta donde me es posible trato de hacer, pensar, decir, siempre obrando desde principios filosóficos. La filosofía me ha hecho ser lo que soy. 


\section{Referencias}

Gómez-Castro, Santiago. (1996). Crítica de la razón latinoamericana. Barcelona: Puvill Libros.

Herrera Restrepo, D. (1970). Hombre y filosofía. Cali: Universidad del Valle.

Herrera Restrepo, D. (1975). La filosofía en Colombia. Bibliografía 1620-1973. Cali: Universidad del Valle.

Herrera Restrepo, D. (1986). Escritos sobre fenomenología. Bogotá: Biblioteca Colombiana de Filosofía.

Herrera Restrepo, D. (2003) Nuestra responsabilidad social. Una reflexión a partir de Husserl. En Acta fenomenológica latinoamericana. Vol. I (pp. 37-48). Lima: Pontificia Católica del Perú.

Herrera Restrepo, D. (2009). Por los senderos del filosofar. Bogotá: Universidad de San Buenaventura.

Husserl, E. (1991). La crisis de las ciencias europeas y la fenomenología trascendental. (Jacobo Muñoz y Salvador Mas, trad.). Barcelona: Crítica.

Rocha de la Torre, A. (Comp.). (2008). La responsabilidad del pensar. Barranquilla: Ediciones Uninorte.

Salazar, R. (1986) Daniel Herrera o la fenomenología como ciencia de la ambigüedad. En Escritos sobre fenomenología. Bogotá: Biblioteca Colombiana de Filosofía. 